\title{
Friture profonde : les interactions huile-produit
}

\author{
Francis COURTOIS \\ Aman MOHAMMAD ZIAIIFAR \\ Isabelle TREZZANI \\ Gilles TRYSTRAM \\ AgroParisTech, \\ INRA UMR1145 Ingénierie-Produits- \\ Aliments, \\ 91300 Massy, \\ France \\ <francis.courtois@agroparistech.fr>
}

La friture profonde peut être définie comme une cuisson par immersion dans une huile ou une matière grasse comestible à une température au-dessus du point d'ébullition de l'eau. C'est un procédé rapide de transfert simultané de chaleur et de matières. II peut être utilisé comme une opération de séchage. Au-delà des applications alimentaires, ce procédé est utilisé pour sécher, pour stabiliser, des carcasses de viande, des boues d'épuration ou encore du bois. La friture profonde reste une opération complexe à cause des deux transferts de matière de direction opposée au sein du matériau : pour les produits à base d'amidon, de l'eau et quelques solutés s'échappent du produit et l'huile entre dans l'aliment. Parfois, même de la matière grasse peut s'échapper du produit vers le bain d'huile. C'est le cas pour des matériaux présentant un taux de matière grasse significatif tels que la viande ou le poisson (Oroszvari et al., 2005). De plus, pendant la friture, le matériau est soumis à des transformations chimiques et physiques à haute température. Finalement, à partir d'un produit brut, la friture combine déshydratation et cuisson, avec la gélatinisation de l'amidon, la dénaturation des protéines, I'aromatisation et la coloration via les réactions de Maillard et l'entrée d'huile. Ces réactions sont fortement

\begin{abstract}
Deep-fat frying process: oil-product interactions
Among the many thermal processes used to prepare tasty food, deep fat frying is one of the fastest and cheapest. Oil serves both as an ingredient and as a heating medium. Recently, nutrition became a major health issue especially in developed countries where obesity is widespread. Fried products being often considered as too calorific, many research projects try to understand the underlying mechanisms of oil uptake in the fried product. There is a difficult challenge in reducing fat content without altering texture and taste. This papers aims at reviewing most interesting researches published on oil uptake.
\end{abstract}

Key words: deep-fat frying, process, oil uptake, product properties, water loss

couplées mais une transformation peut être privilégiée en adaptant et optimisant les paramètres du procédé. Actuellement, la prise d'huile est considérée comme le point critique majeur, d'un point de vue nutritionnel, en friture profonde. Ceci est dû à l'épidémie d'obésité qui prévaut dans les régions développées et même dans les régions en développement où les repas les plus économiques sont riches en matières grasses et en sucre (FAO, 2002). Le défi majeur est donc d'améliorer le procédé de friture en contrôlant et, surtout, diminuant le taux de matière grasse finale du produit frit. De plus, pendant la friture, des molécules potentiellement toxiques apparaissent au sein du bain d'huile comme une conséquence de la détérioration de I'huile en présence d'oxygène, de chaleur et d'eau. Ces composés modifient les propriétés physico-chimiques de l'huile et peuvent favoriser la prise d'huile. Ainsi, pour cette deuxième raison, la qualité et la quantité d'huile entrant dans le produit doivent être contrôlées sérieusement. De nombreuses études sur ce sujet ont été publiées ces dernières années afin de mieux comprendre ce phénomène. II semble que les mécanismes de l'entrée d'huile ont été largement mis à jour mais de nombreuses contradictions subsistent du fait des nombreux paramètres d'influence (Ziaiifar et al., 2008).

\section{Aspects nutritionnels}

Les produits frits sont d'une grande variété. $\mathrm{Si}$ on considère les produits à base d'amidon, deux produits prédominent : les frites de pommes de terre ou de plantain où la déshydratation est seulement partielle. L'épaisseur du produit aura une grande influence. En général, les produits de faible épaisseur présentent une teneur en eau très faible. Ils peuvent donc être conditionnés facilement pour la conservation et la distribution. Cependant comme les produits fins présentent un taux de matière grasse élevée (30 à $40 \%$ ), la stabilité de l'huile pendant la conservation doit être gérée avec précaution pour éviter le développement d'arômes indésirables (rancidité) dans le produit. Les produits épais, quant à eux, présentent une teneur en eau intermédiaire d'à peu près 30 à $50 \%$ mais avec une faible teneur en matière grasse (inférieure à $15 \%$ ). Typiquement, ils ont une croûte croustillante et un cœur moelleux comme de la pomme de terre cuite.

Ces produits peuvent être obtenus à partir d'ingrédients frais ou surgelés, ayant souvent subi une préfriture. Cette préfriture consiste généralement en une

Pour citer cet article : Courtois F, Mohammad Ziaiifar A, Trezzani I, Trystram G. Friture profonde : les interactions huile-produit. OCL 2012 ; $19(2)$ : 89-95. doi : 10.1684/ocl.2012.0444 
friture rapide pour éviter toute action enzymatique avant l'étape de surgélation. Une deuxième étape de friture est ensuite nécessaire pour achever la cuisson. Dans ces produits, le taux de matière grasse final est généralement plus élevé que dans des produits frits directement du fait des deux étapes de friture successives. Par ailleurs, il a été démontré que, pendant la friture certains micronutriments hydrosolubles (acide ascorbique et potassium) du produit frit sont préservés (Rojas et al., 2006). Ainsi, une opération à haute température et temps court est intéressante pour la conservation de certaines propriétés nutritionnelles.

Le résultat des réactions chimiques complexes de thermo-oxydation et d'isomérisation est une quantité accrue de composés lipidiques dégradés dans le bain d'huile tels que acides gras libres, mono et diglycérides, polymères, composé trans, composés carbonylés potentiellement toxiques, etc., qui seront absorbés ensuite par le produit, réduisant sa qualité au niveau nutritionnel, organoleptique et sanitaire (Gertz, 2005). Même si une réglementation existe pour limiter les composés polaires à $25 \%$ et la teneur en polymères à $12 \%$, des composés potentiellement toxiques peuvent apparaître dans le bain d'huile. Les principaux composés actuellement identifiés comme potentiellement cancérigènes sont des carbonyles ou mono époxydes et quelques aldéhydes produits à partir de l'acide linoléique. Ainsi le 4-hydroxy-2-transnonenal a été montré comme étant cytotoxique (Seppanen et Saari Csallany, 2002 ; Matthaus et Wohrmann, 2006).

Afin d'assurer la consommation de produits de friture sains, des huiles de friture doivent donc être choisies sur la base d'un compromis entre leur stabilité thermique, leurs propriétés nutritionnelles et leur coût. Les principaux types d'huiles à l'échelle mondiale sont I'huile de soja, I'huile de palme, I'huile de canola, I'huile de tournesol, I'huile de graines de coton, I'arachide, I'huile de palmiste, de noix de coco, d'olive, les huiles de maïs etc. Parmi elles, les huiles de soja, palme, canola et les huiles de tournesol représentent $80 \%$ de la production mondiale (AAC, 2005). L'huile de palme a un niveau élevé $d^{\prime}$ acides gras saturés, bienvenus de par leur stabilité thermique, mais pas intéressants d'un point de vue nutri- tionnel. D'autre part, les huiles présentant une teneur élevée en acides gras polyinsaturés tels que le soja, le canola, et les huiles de tournesol sont nutritionnellement intéressantes, mais sensibles à I'oxydation. En outre, les huiles linoléiques comme I'huile de tournesol et de soja devraient être évitées en raison du risque de production de 4hydroxy-2-trans-nonénal. Les huiles insaturées peuvent être partiellement hydrogénées afin d'accroître leur stabilité chimique. Toutefois, les acides gras trans sont un sous-produit majeur de l'hydrogénation qui peut représenter $30 \%$ du total des acides gras. Des études épidémiologiques ont démontré que la consommation d'acides gras trans peut être corrélée avec le risque de maladie cardiovasculaire (Daniel et al., 2005). Par conséquent, les huiles de friture idéales peuvent être un mélange de différentes huiles présentant des propriétés chimiques complémentaires ou peuvent être produites à partir de plantes améliorées comme le tournesol riche en acide oléique, et le canola riche en acide oléique. Ces huiles ont une bonne qualité nutritionnelle pour la friture, sont plus chères mais peuvent répondre à des spécifications santé plus exigeantes.

\section{Localisation de I'huile absorbée}

L'absorption d'huileestprincipalementun phénomène de surface comme le confirment les observations expérimentales. II a été ainsi prouvé par calorimétrie (Aguilera et Gloria, 1997) que la croûte des frites contenait environ 6 fois plus d'huile que l'intérieur. Keller et al. (1986) ont pu visualiser directement la distribution de I'huile dans des frites en utilisant un colorant. Ils ont conclu que I'huile ne pénétrait pas plus profondément que les premières couches cellulaires. Ce résultat a été confirmé en microscopie électronique à balayage par Lisinska et Golubowska (2005). Cette technique a permis par ailleurs d'étudier la structure macro poreuse hétérogène qui se développe dans le produit pendant la friture.

Des mesures au microscope confocal réalisées par Pedreschi et al. (1999) ont montré que I'huile enveloppe la surface des cellules de pomme de terre et est principalement prise au piège dans les vides intercellulaires générés pendant la coupe. L'huile ne pénètre pas dans les cellules intactes en raison de la composition du parenchyme de la pomme de terre. En effet, la paroi cellulaire est plus épaisse et plus solide que la lamelle intermédiaire partagée avec les cellules environnantes. Par conséquent, c'est une séparation des cellules qui se produit plutôt qu'une rupture des cellules. Aguilera et al. (2001) proposent des explications complémentaires suite à des observations microscopiques in situ des cellules isolées de pommes de terre pendant la cuisson. Ils expliquent que lors de la friture, la gélatinisation et le gonflement de l'amidon se produisent très rapidement, rendant ainsi les cellules de pommes de terre capables de bien supporter une déshydratation et un retrait sans générer de rupture.

\section{Période d'absorption de I'huile}

Les premières observations expérimentales de Ufheil et Esher (1996) sur des tranches de pommes de terre ont montré que l'absorption d'huile s'est produite pendant le refroidissement. Ils ont effectué des expériences successives ajoutant de I'huile de friture colorée au bain d'huile à différents moments de la friture et quantifié la quantité d'huile colorée dans les frites avec une méthode combinant extraction et réfractométrie. Ils ont constaté que même si I'huile colorée est ajoutée seulement au cours de la dernière période de la friture, la proportion d'huile colorée dans le produit frit est encore très élevée. Par conséquent, I'huile ne pénètre pas dans les frites pendant la friture, mais est absorbée par la couche superficielle d'huile lorsque le produit est retiré de la friteuse du fait de la dépressurisation du produit générée par la condensation soudaine de la vapeur d'eau présente dans les pores. Cependant, certains auteurs pensent que l'absorption d'un peu d'huile a lieu lors de la friture. Bouchon et al. (2003) ont noté qu'une petite quantité d'huile qu'ils ont appelée "huile structurelle » (en opposition à I'huile adhérant à la surface ou de I'huile aspirée pendant le refroidissement), pourrait être absorbée pendant la friture. Cette petite quantité pourrait dépendre de la vitesse de séchage et de la durée totale de la friture. En outre, selon Saguy et Pinthus (1995), lorsque la vitesse de déshydratation diminue pendant la 
friture, le taux d'absorption d'huile augmente du fait de la réduction de la pression de vapeur interne des pores. Mehta et Swinburn (2001) ont montré qu'un peu de matière grasse était absorbée lorsque le produit est immergé alors que de la vapeur est toujours en train de s'échapper. En accord avec leurs observations, la figure 1 montre les résultats expérimentaux de distribution $d^{\prime}$ huile pendant la friture et le refroidissement d'une tranche de pomme de terre. Pendant la cuisson, une petite quantité d'huile est absorbée par la pomme de terre, alors que pendant le refroidissement, la teneur en huile interne augmente à un rythme rapide pendant la première minute de refroidissement tandis que I'huile de surface diminue jusqu'à ce qu'un équilibre soit atteint après $4 \mathrm{~min}$ de refroidissement.

\section{Mécanismes d'absorption d'huile}

L'absorption d'huile est un mécanisme complexe qui n'est pas encore clairement compris. La structure initiale du produit, les échanges divers entre le produit et le fluide de chauffage et les variations des propriétés des produits et des huiles sont des facteurs qui compliquent l'étude. La plupart des auteurs conviennent que pendant la friture, les transferts de chaleur et de masse sont gouvernés par le transfert de chaleur à la surface du produit. La vitesse de vapo-

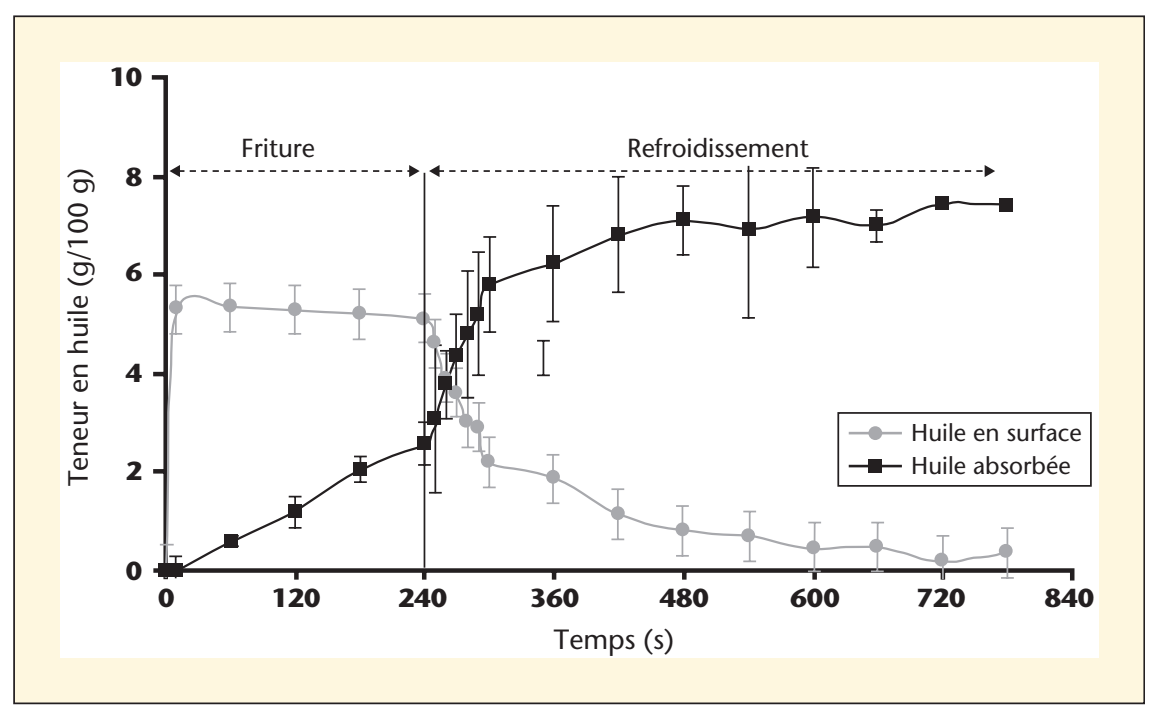

Figure 1. Teneur en huile absorbée ou restée à la surface de frites lors de la friture $\left(170^{\circ} \mathrm{C}\right)$ et du refroidissement $\left(20^{\circ} \mathrm{C}\right.$ ) [la discrimination de la teneur en huile absorbée et de surface en utilisant la méthode Moreira et al. (1997) avec de l'éther de pétrole].

risation est proportionnelle à la différence de température entre I'huile et le point d'ébullition de l'eau. Farkas et al. (1996) furent les premiers scientifiques à proposer une description physique complète de la friture. Ils ont considéré que ce procédé doit être décrit comme un problème complexe de Stephan du fait des transferts couplés de chaleur et de matière entraînant le déplacement d'un front de vaporisation mobile qui sépare deux régions dynamiques: une croûte déshydratée et un noyau humide. Comme la croûte présente une faible conductivité thermique, elle ralentit le transfert de chaleur et est en partie responsable de la diminution de la vitesse de déshydratation. En général, on peut dire que plus l'eau est retirée de la surface, plus I'huile est absorbée. Dans la figure 2, la relation entre la teneur en huile et la perte d'humidité est représentée. En effet, Gamble et al. (1987) ont constaté que la perte d'humidité et l'absorption d'huile étaient liées entre elles et étaient toutes deux des fonctions linéaires de la racine carrée du temps de friture. Ils ont fait I'hypothèse que I'huile entrant dans la tranche se situerait dans les vides laissés par l'eau qui s'échappe. Par conséquent, en plus des aspects quantitatifs, la perte d'eau peut devenir une variable explicative de la transformation et l'absorption d'huile en particulier parce que l'eau d'évacuation est à l'origine de phénomènes physiques très divers tels que la création de porosité (Vitrac, 2000).
Ainsi, la déshydratation se produisant à une température supérieure à $100{ }^{\circ} \mathrm{C}$, la vapeur d'eau, pour s'échapper du produit, doit trouver les zones de faiblesse dans I'adhérence cellulaire. Ceci conduit à la formation de cheminements capillaires accroissant la porosité de surface. En outre, une partie de cette vapeur peut être piégée dans les pores du fait de la diffusion intercellulaire limitée, puis se dilater et se surchauffer, déformant les parois des pores et contribuant ainsi à la porosité du produit. En conséquence, certaines études ont examiné l'augmentation de la porosité lors de la friture et I'ont corrélée à la quantité d'huile absorbée (Pinthus et al., 1995 ; Moreira et al., 1997 ; Ziaiifar et al., 2010). La caractérisation de la microstructure des produits apparaît ainsi comme un facteur déterminant dans la description des transferts à l'échelle macroscopique, en particulier pour l'absorption d'huile.

\section{Facteurs influant I'absorption d'huile}

\section{Propriétés du produit}

Comme la prise d'huile est un phénomène de surface, le rapport entre la surface d'échange huile-produit et le volume du produit va déterminer la quantité d'huile qui peut être absorbée. Les résultats montrent que l'absorption d'huile augmente considérablement lorsque l'épaisseur du produit est réduite et la surface du produit est augmentée (Guillaumin, 1983). Par exemple, les frites absorbent moins d'huile que les chips en raison d'un plus petit rapport surface/volume (Paul et Mittal, 1997). Une relation linéaire a été établie entre la surface et la teneur en huile (Gamble et Rice, 1988). Comme l'essentiel de la matière grasse pénètre le produit à travers les pores de la croûte, les propriétés structurales de la couche externe de l'aliment sont importantes. En effet, les cellules rompues lors de la coupe sont un lieu privilégié pour l'absorption d'huile (Saguy et Pinthus, 1995). L'utilisation de lames de coupe de qualité peut donc diminuer la rugosité de surface du produit et donc la surface, diminuant l'absorption d'huile.

Le taux de matière sèche initial du produit est un facteur qui influe sur l'absorption d'huile pendant la friture à cause de la relation entre la perte d'eau et l'absorption d'huile (Kozempel et al., 


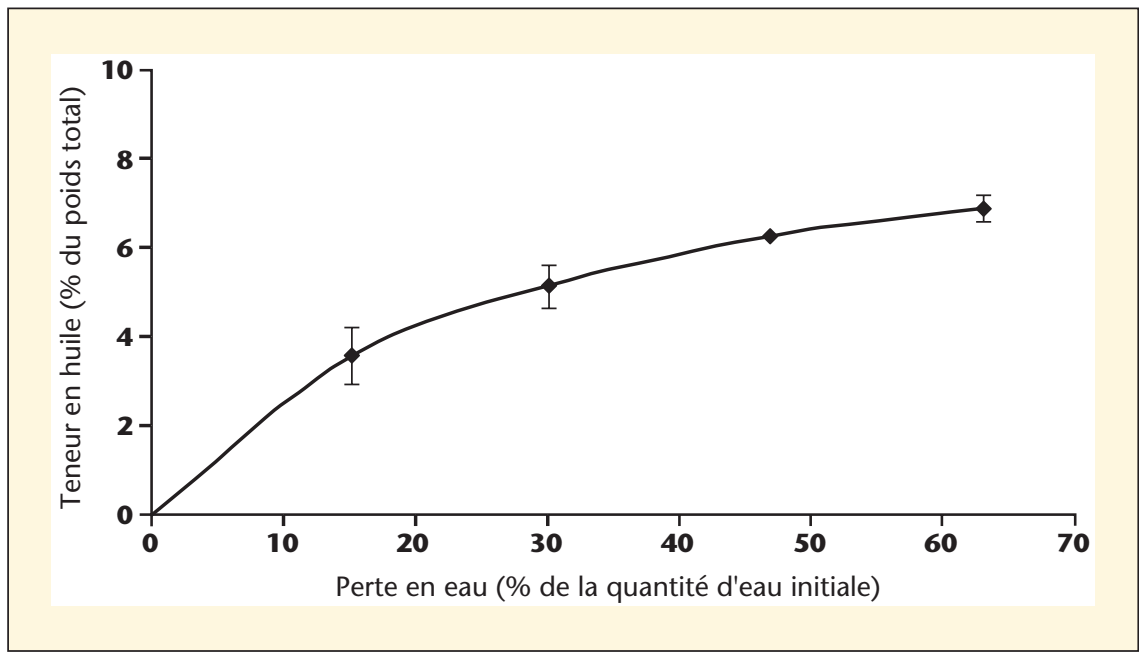

Figure 2. Teneur en huile vs teneur en eau des frites durant une friture à $155^{\circ} \mathrm{C}$ (Ziaiifar et al., 2008).

1991 ; Pinthus et Saguy, 1994 ; Moreira et al., 1997 ; Krokida et al., 2001 ; Yamsaengsung et Moreira, 2002). En effet, pour un produit final frit qui présente une teneur en eau intermédiaire (produit épais) tels que des frites de plantain, de pomme de terre ou cubes $d^{\prime}$ aubergine, plus la teneur en eau initiale est élevée, plus élevée est la prise d'huile finale (tableau 1). Les données du tableau 1 montrent également que la perte d'humidité importante qui se produit lors de la friture des produits minces (chips) conduit à une absorption de graisse considérable en raison des vastes volumes vides laissés par le départ de l'eau (Gamble et al., 1987). Toutefois, le comportement atypique de l'aubergine pendant la friture doit être remar- qué. En effet, même si la teneur en eau résiduelle est encore assez élevée après la friture (seulement $50 \%$ de l'eau initiale est retirée), la prise d'huile est aussi élevée que dans les produits totalement déshydratés comme les frites. Ce phénomène peut être expliqué par le fait que l'aubergine est un produit nonféculent dont la structure est très fragile et spongieuse (Kalogeropoulos et al., 2006).

\section{Choix de l'huile de friture}

$L^{\prime}$ 'effet du type d'huile est très différent en fonction de l'auteur. Kita et Lisinska (2005) écrivent que l'absorption de I'huile est plus élevée lorsque la quantité d'acides gras insaturés dans I'huile augmente. À l'opposé, Vitrac (2000) a montré que l'absorption d'une huile insaturée comme l'huile de coton est plus faible que celle de I'huile de palme en raison de sa faible viscosité au cours du refroidissement et de sa capacité à s'égoutter facilement. Ces contradictions pourraient s'expliquer par le fait que la viscosité d'une huile est très influente dans le mécanisme de son absorption, mais elle contribue à la fois à I'adhésion et au drainage. En outre, I'huile de friture peut contenir une partie de la graisse qui se solidifie en refroidissant et être plus difficile à drainer hors de l'aliment tout en étant moins susceptible de pénétrer profondément dans les pores de la croûte. Plus la viscosité initiale de l'huile est élevée et plus sa migration est ralentie. La viscosité de I'huile ne dépend pas seulement du type d'huile, mais aussi de la température et de la qualité de cette même huile. La viscosité de I'huile diminue avec une température décroissante suivant l'équation d'Arrhenius. La tension superficielle initiale est également un facteur important à considérer dans les mécanismes capillaires menant à l'absorption d'huile. Une augmentation de la tension interfaciale conduit à une augmentation de I'absorption d'huile. Par conséquent, I'ajout de tensio-actifs (agents tensioactifs ou agents mouillants) tels que Tween 80 (polysorbate) et Span80 (sorbitane) dans l'huile de friture pourrait modifier les propriétés de surface et la teneur en huile dans le produit final (Pinthus et Saguy, 1994). Cependant,

Tableau 1. Teneurs en eau et en matière grasse de divers produits alimentaires.

\begin{tabular}{|c|c|c|c|c|}
\hline & Teneur en eau & Teneur en huile & Teneur en eau & Teneur en huile \\
\hline Produits non frits & (g/100 g frais) & (g/100 g frais) & $\left(\mathbf{k g} / \mathbf{k g}^{*}\right)$ & $\left(\mathbf{k g} / \mathbf{k g}^{*}\right)$ \\
\hline Plantain (Rojas et al., 2006) & 60 & 0,1 & 1,53 & 0,005 \\
\hline Pomme de terre (Talburt, 1987) & 80 & 0,1 & 4 & 0,005 \\
\hline Aubergine (Kalogeropoulos et al., 2006) & 95 & 0,1 & 19,38 & 0,005 \\
\hline Manioc (Vitrac, 2000) & 60 & 0,1 & 1,5 & 0,005 \\
\hline Farine de maïs (Moreira et al., 1999) & 47 & 1,5 & 0,88 & 0,03 \\
\hline \multicolumn{5}{|l|}{ Produits frits } \\
\hline Frites de plantain (Rojas et al., 2006) & 32 & 7 & 0,8 & 0,15 \\
\hline Frites de pomme de terre (Talburt, 1987) & 44 & 13 & 1,05 & 0,3 \\
\hline Chips de pomme de terre (Talburt, 1987) & 2 & 40 & 0,03 & 0,69 \\
\hline Cubes d'aubergine (Kalogeropoulos et al., 2006) & 50 & 40 & 3,85 & 2,71 \\
\hline Chips de manioc (Vitrac, 2000) & 2 & 25 & 0,05 & 0,35 \\
\hline Chips de maïs-tortilla (Moreira et al., 1999) & 2 & 25 & 0,025 & 0,35 \\
\hline
\end{tabular}

* kg par kg de matière sèche déshuilée 
ces produits ne sont pas largement approuvés pour un usage alimentaire.

Le vieillissement de I'huile résulte aussi en une augmentation de la viscosité à la suite de la formation de polymères et la diminution de l'angle de contact à cause de la formation de composés polaires. L'augmentation de la viscosité pourrait contribuer à une augmentation de la quantité d'huile sur la surface de I'aliment, tandis que la diminution de I'angle de contact pourrait augmenter les propriétés de mouillage de l'huile, les deux résultant en une teneur en huile plus élevée. Pour cette raison, Tseng et al. (1996) ont fait valoir que l'huile de surface sur les chips a augmenté avec la dégradation de I'huile. Pourtant, certaines études prouvent que la teneur en huile totale finale des chips n'est pas significativement affectée par la qualité de I'huile après de nombreux cycles de friture (Mehta et Swinburn, 2001). Néanmoins, cette évolution chimique qui peut avoir des effets néfastes sur la quantité et la qualité d'huile peut être ralentie par l'addition d'antioxydants naturels ou synthétiques (Che Man et Jaswir, 2000 ; Houhoula et al., 2004).

\section{Paramètres procédé}

Un large éventail de facteurs, y compris les conditions des prétraitements, la friture à proprement dite et les posttraitements sont connus pour influencer l'absorption d'huile dans les aliments. Certains de ces éléments et leurs paramètres ont été brevetés en tant que moyens de diminuer l'absorption d'huile.

\section{Prétraitements}

Les méthodes les plus populaires à l'échelle industrielle sont le blanchiment, le séchage à l'air, la déshydratation osmotique, la cuisson à la vapeur, et le traitement de surface (enrobage). Le blanchiment est un processus de préparation des aliments, où la substance alimentaire est plongée dans l'eau bouillante ou la vapeur afin d'inactiver les enzymes et micro-organismes présents. L'effet sur I'absorption de I'huile est assez ambigu en raison des conditions différentes appliquées. Certains auteurs (Rimac-Brncic et al., 2004) affirment que le blanchiment avant la friture diminue la teneur en matière sèche du produit en raison de la migration des composants hydrosolubles du produit vers l'eau de blanchiment. Par conséquent, ce phénomène augmente la teneur en eau et donc la teneur en huile (Alvarez et al., 2000 ; Pedreschi et al., 2005). D'autre part, comme l'ont souligné d'autres études, la gélatinisation de l'amidon de surface qui se produit pendant le blanchiment pourrait former une couche mince ferme qui protège les aliments de l'absorption d'huile (Califano et Calvelo, 1987). En outre, le blanchiment peut activer les enzymes pectinestérase qui peuvent causer l'effondrement des parois des cellules de surface, entraînant une diminution de la porosité et de la teneur en huile du produit (Aguilera-Carbo et al., 1999).

Le pré-séchage est un autre moyen utilisé pour créer une matrice sèche très solide autour du produit. Cette technique diminue la teneur en eau totale du produit et limite l'absorption d'huile (Lamberg et al., 1990 ; Moreira et al., 1999 ; Vitrac, 2000). En outre, le retrait qui se produit pendant le séchage réduit la surface totale et diminue par conséquent le transfert de masse. Enfin, ce procédé est intéressant à deux titres : il diminue l'absorption d'huile, tout en améliorant les propriétés organoleptiques du produit en augmentant son croquant (Debnath et al., 2003). Semblable au séchage convectif, la déshydratation osmotique, décrite comme une déshydratation partielle par immersion du produit brut dans une solution concentrée (habituellement sucrée ou salée), diminue la teneur en huile en diminuant la teneur en eau initiale du produit. Krokida et al. (2001) a montré que les frites trempées dans une solution de sucre (40\% en masse) ont présenté une réduction de $60 \%$ de la teneur en matières grasses et, en trempant dans du $\mathrm{NaCl}$ (20\% en masse) et dans des solutions de maltodextrine (20\% en masse), pour le même temps de traitement, on peut obtenir des réductions de la teneur en huile de $35 \%$ et $15 \%$, respectivement.

Enfin, la cuisson à la vapeur, comme le blanchiment et le séchage à l'air, peut être utilisée pour former une barrière étanche à la surface extérieure du produit en raison d'une sévère gélatinisation de l'amidon. Cette couche extérieure présente une forte résistance à l'entrée d'huile dans le produit. Par conséquent, la teneur en huile interne finale est plus faible dans ces produits et l'essentiel de l'huile reste à la surface du produit (Rajkumar et al., 2003).
L'enrobage est une autre technique de préfriture qui consiste à plonger un produit brut dans une suspension pendant une courte période immédiatement avant qu'il soit frit. Ce traitement de surface réduit la porosité de la surface et crée également une barrière contre I'absorption d'huile. En effet, l'enrobage diminue non seulement l'absorption de graisse, mais aussi la perte d'eau pendant la friture. Les propriétés les plus importantes pour de bons hydrocolloïdes sont la capacité de formation d'un film, la stabilité à la chaleur, les propriétés de transfert d'eau et de lipides, I'aspect organoleptique et les qualités nutritionnelles. Certains des hydrocolloïdes les plus couramment utilisés et efficaces sont dérivés de la cellulose comme I'hydroxypropylcellulose (HPC), la méthylcellulose (MC), et I'hydroxypropylméthylcellulose (HPMC) (Albert et Mittal, 2002). L'enrobage semble être une méthode efficace pour réduire l'absorption de l'huile : certaines études expérimentales ont démontré que l'absorption d'huile pourrait être réduite de $40 \%$ dans les frites en utilisant des enrobages de cellulose avant de les frire, bien que leur teneur en eau soit plus élevée (Garcia et al., 2002 ; Mellema, 2003).

\section{Température de I'huile}

\section{et le couple temps de friture.}

La température de friture dépend du type de produit, de sa taille et de sa composition. Elle varie souvent de 120 à $190{ }^{\circ} \mathrm{C}$. Des températures élevées $\mathrm{d}^{\prime}$ huile $\left(160-190{ }^{\circ} \mathrm{C}\right.$ ) peuvent permettre le transfert rapide de la chaleur, un brunissement rapide et un temps de cuisson court. Pour cette raison, mettre une trop grande quantité de nourriture froide dans I'huile chaude est préjudiciable à la qualité des produits et à l'efficacité du procédé, car cela entraîne une diminution spectaculaire de la température de I'huile et un temps de cuisson plus long. A l'échelle industrielle, une bonne charge en produit pour I'huile est généralement dans un ratio de 1/6 (Mehta et Swinburn, 2001). Une augmentation de la température de I'huile déclenche une augmentation des vitesses de déshydratation et de réaction couplées. Par conséquent, des températures élevées vont limiter le temps de friture. Toutefois, pour la même teneur résiduelle, l'effet de la température de friture est marginal et certains auteurs avancent même que 
les températures comprises entre 140 et $190{ }^{\circ} \mathrm{C} \mathrm{n}^{\prime}$ ont pas d'influence sur l'absorption d'huile (Gamble et Rice, 1987 ; Moreira et al., 1997).

Toutefois, I'idée de limiter la température d'huile afin de limiter sa dégradation a motivé l'étude de températures de friture inférieures à $140{ }^{\circ} \mathrm{C}$. Ces travaux ont montré qu'une température telle que $120{ }^{\circ} \mathrm{C}$ nécessite un temps de friture plus long et engendre finalement une absorption d'huile plus élevés, pour une même teneur en eau résiduelle (Talburt, 1987 ; Pedreschi et Moyano, 2005 ; Moyano et Pedreschi, 2006; Rojas et al., 2006). Ce phénomène peut être expliqué par le temps de friture allongé et le flux évaporatoire plus faible de l'eau en surface ou par le développement de structures différentes (porosité en particulier). En effet, lors de la friture à une température $d^{\prime}$ 'huile très faible, telle que $120^{\circ} \mathrm{C}$, la croûte est plus souple et pourrait laisser I'huile pénétrer facilement dans le produit (Blumenthal et Stier, 1991).

La friture sous vide permet quant à elle de fonctionner à une concentration en oxygène inférieure et à une température inférieure (sous vide la température d'ébullition est très basse). Ce procédé peut donc conserver les couleurs naturelles et les saveurs et limiter la dégradation d'huile. Ainsi, Garayo et Moreira (2002) ont démontré que la teneur en huile finale est inférieure pour les frites frites sous vide pour une même déshydratation. Plus récemment, Liu Ping et al. (2005) ont affirmé que la perte d'humidité et l'absorption d'huile augmentent tandis que le degré de dépression augmente pour les chips de carottes et Tan et Mittal (2006) ont trouvé que l'absorption d'huile est plus élevée dans les beignets frits sous vide que ceux frits à la pression atmosphérique pour une humidité finale identique. En conclusion, la friture sous vide a été insuffisamment étudiée et son effet sur l'absorption de l'huile n'est pas clairement connu.

\section{Post-traitements}

Lorsque le produit est retiré du bain de friture, la température commence immédiatement à diminuer. En dessous de $100{ }^{\circ} \mathrm{C}$, la vapeur d'eau se condense et provoque une chute de pression interne, ce qui entraîne une différence de pression favorisant l'entrée d'huile. La température influe sur la viscosité et la tension interfaciale qui sont impliquées dans les phénomènes d'absorption d'huile. Matz (1993) a affirmé que si le produit est retiré de la friteuse alors que sa température est toujours en augmentation, I'absorption de l'huile sera plus faible. L'hydrodynamique de refroidissement sont également importants : en agitant vigoureusement le panier de produits frits immédiatement après le retrait de la friteuse, on peut drainer I'huile de surface si elle est encore liquide et n'a pas encore été aspirée dans les pores. L'huile qui serait en mesure de pénétrer dans les pores est donc limitée dans ce cas (Thanatuksorn et al., 2005).

On peut combiner cela avec un séchage (à I'air ou à la vapeur d'eau surchauffée) après friture pour réduire la teneur en huile finale en réduisant le temps de contact entre le produit et l'huile (Topin et Tadrist, 1997 ; Li et al., 1999). Par exemple, Myers et Loewe (1990) ont rapporté une technique de retrait prématuré du produit à haute teneur en humidité suivie d'un séchage ultérieur à l'aide de vapeur surchauffée. Cette combinaison a entraîné une réduction de 30 \% de l'absorption d'huile selon eux. Toutefois, le principal inconvénient du séchage à la vapeur surchauffée est sa température élevée pouvant causer des dommages aux produits thermosensibles. Enfin, une finition aux micro-ondes après la friture peut aussi être une solution de rechange pour obtenir une faible teneur en huile (Blau et al., 1965). Cependant, cette technique est difficile à introduire à l'échelle industrielle en raison de difficultés à le contrôler.

\section{Conclusion}

La friture, et plus particulièrement l'interaction entre l'huile et le produit, constitue une thématique de recherche riche et très active. Si l'opération unitaire est simple à comprendre, les phénomènes sous-jacents sont particulièrement complexes et font l'objet parfois de conclusions contradictoires. Le challenge reste entier : des produits frits croustillants, gouteux et optimisés sur le plan nutritionnel.

\section{RÉFÉRENCES}

AAC, Agriculture and Agri-Food Canada. Huiles végétales : un marché concurrentiel en évolution. Le Bulletin bimensuel $2005 ; 18$ : 1494-813.
Aguilera JM, Gloria H. Determination of oil in fried potato products by differential scanning calorimetry. J Agric Food Chem 1997 ; 45 : 781 85.

Aguilera JM, Cadoche L, Lopez C, Gutierrez G. Microstructural changes of potato cells and starch granules heated in oil. Food Res Int 2001 ; 34 : 939-47.

Aguilera-Carbo A, Cesar-Montanez J, Anzaldua-Morales A, de la Luz-Reyes M, ContrerasEsquivel J, Noe-Aguilar C. Improvement of color and limpness of fried potatoes by in situ pectinesterase activation. Eur Food Res Technol $1999 ; 210$ : 49-52.

Albert S, Mittal GS. Comparative evaluation of edible coatings to reduce fat uptake in a deep fried cereal product. Food Res Int 2002 ; $35: 445-58$.

Alvarez MD, Morillo MJ, Canet W. Characterization of the frying process of fresh and blanched potato strips using response surface methodology. Eur Food Res Technol 2000 ; 211 : 326-35.

Blau R, Powell M, Gerling JE. Megacycle microwave treatments in potato chip finishing. Processing, Production and Technology division Meetings. Potato Chip Institute International $1965: 1-8$.

Blumenthal MM, Stier RF. Optimization of deep-fat frying operations. Trends Food Sci Tech $1991 ; 2$ : 144-8.

Bouchon P, Aguilera JM, Pyle DL. Structure oil absorption relationships during deep-fat frying. J Food Sci 2003 ; 68 : 2711-6.

Califano AN, Calvelo A. Adjustment of surface concentration of reducing sugars before frying of potato strips. / Food Proces Preserv 1987, 12, 1-9.

Che Man YB, Jaswir I. Effect of rosemary and sage extracts on frying performance of refined, bleached and deodorized (RBD) palm olein during deep-fat frying. Food Chemistry $2000 ; 69$ : 301-7.

Daniel DR, Thompson LD, Shriver B], Wu CK, Hoover LC. Nonhydrogenated cottonseed oil can be used as a deep fat frying medium to reduce trans-fatty acid content in French fries. I Am Dietetic Assoc 2005 ; $105: 1927-32$.

Debnath S, Bhat KK, Rastogi NK. Effect of predrying on kinetics of moisture loss and oil uptake during deep fat frying of chickpea flour-based snack food. Lebensmittel-Wissenschaft und-Technologie $2003 ; 36$ : 91-8.

FAO. The developing world's new burden: obesity. http ://www.fao.org/FOCUS/E/obesity/obes1.htm. 2002.

Farkas BE, Singh RP, Rumsey TR. Modeling heat and mass transfer in immersion frying. I. Model development. J Food Eng 1996 ; 29 : 211-26. 
Gamble MH, Rice P. Effect of pre-fry drying of oil uptake and distribution in potato crisp manufacture. Int J Food Sci Technol 1987 ; 22 : 535-48.

Gamble MH, Rice P. The effect of slice thickness on potato crisp yield and composition. J Food Eng $1988 ; 8$ : 31-46.

Gamble MH, Rice P, Selman JD. Relationship between oil uptake and moisture loss during frying of potato slices from c.v. Record U. K. tubers. Int J Food Sci Techno/ 1987; 22: 233-41.

Garayo J, Moreira R. Vacuum frying of potato chips. J Food Engin 2002 ; 55 : 181-91.

Garcia MA, Ferrero C, Bertola N, Martino M, Zaritzky N. Edible coatings from cellulose derivatives to reduce oil uptake in fried products. Innovative Food Science and Emerging Technologies 2002 ; 3 : 391-7.

Gertz C. Quality control and safety of cooking oils. Frankfurt : Workshop Deep-Frying. 2005.

Guillaumin R. Animal fats in the food industry. V. Desirable characteristics for frying. Revue Française des Corps Gras 1983 ; 30 : 347-54.

Houhoula DP, Oreopoulou V, Tzia C. Antioxidant efficiency of oregano in frying and storage of fried products. Eur J Lipid Sci Technol $2004 ; 106:$ 746-51.

Kalogeropoulos N, Mylona A, Chiou A, loannou MS, Andrikopoulos NK. Retention and distribution of natural antioxidants ([alpha]-tocopherol, polyphenols and terpenic acids) after shallow frying of vegetables in virgin olive oil. LWT - Food Science and Technology 2006 ; 40 : 1008-17.

Keller C, Escher F, Solms J. A method for localizing fat distribution in deep-fat fried potato products. Lebensmittel-Wissenschaft und Technologie $1986 ; 19$ : 346-8.

Kita A, Lisinska G. The influence of oil type and frying temperatures on the texture and oil content of French fries. I Sci Food Agric $2005 ; 85$ : 2600-4.

Kozempel MF, Tomasula PM, Graig JC. Correlation of moisture and oil concentration in French fries. LWT - Food Science and Technology $1991 ; 24$ : 445-8.

Krokida MK, Oreopoulou V, Maroulis ZB, Marinos-Kouris D. Effect of osmotic dehydration pretreatment on quality of French fries. J Food Eng $2001 ; 49$ : 339-45.

Lamberg I, Hallstroem B, Olsson H. Fat uptake in a potato drying/frying process. Lebensmittel-Wissenschaft und Technologie $1990 ; 23: 295-300$.

Li YB, Seyed-Yagoobi J, Moreira RG, Yamsaengsung $R$. Superheated steam impingement drying of tortilla chips. Drying Technology $1999 ; 17$ : 191-213.

Lisinska G \& Golubowska G. Structural changes of potato tissue during French fries production. Food Chemistry $2005 ; 93: 681-7$.
Liu-Ping F, Min Z \& Mujumdar, AS. Vacuum frying of carrot chips. Drying Technology $2005 ; 23: 645-56$.

Matthaus B, Wohrmann D. Formation of 4-hydroxy-2- trans-nonenal during heating of edible oils. 4th Euro Fed Lipid Congress: Oils, Fats and Lipids for a Healthier Future. Madrid, Spain, 2006.

Matz SA. Snack Food Technology. New York : AVI. 1993.

Mehta U, Swinburn B. A review of factors affecting fat absorption in hot chips. Critical Reviews in Food Science and Nutrition 2001 ; $41: 133-54$.

Mellema M. Mechanism and reduction of fat uptake in deepfat fried foods. Trends Food Sci Tech $2003 ; 14$ : 364-73.

Moreira RG, Sun $X$ \& Chen $Y$. Factors affecting oil uptake in tortilla chips in deepfat frying. J Food Eng 1997 ; 31 : 485-98.

Moreira RG, Castell-Perez ME, Barrufet MA. Deep Fat Frying: Fundamentals and Applications. Gaithersburg, MD : Aspen Publisher, 1999.

Moyano PC, Pedreschi F. Kinetics of oil uptake during frying of potato slices: effect of pre-treatments. LWT - Food Science and Technology 2006 ; 39 : 285-91.

Myers MA, Loewe R. Functionality of Hydrocolloids in Batter Coating Systems. Batters and Breading in Food Processing. St Paul, MN : American Association of Cereal Chemists Publication. 1990.

Oroszvari BK, Bayod E, Sjoholm I, Tornberg E. The mechanisms controlling heat and mass transfer on frying of beefburgers. Part 2 : The influence of the pan temperature and patty diameter. J Food Eng 2005 ; 71 : 18-27.

Paul S, Mittal GS. Regulating the use of degraded oil/fat in deep-fat/oil food frying. Critical Review on Food Sciences and Nutrition 1997 ; 37 : 635-62.

Pedreschi F, Moyano P. Effect of pre-drying on texture and oil uptake of potato chips. LWT - Food Science and Technology 2005 ; 38 : 599-604.

Pedreschi F, Aguilera JM, Arbildua J. Use of confocal laser scanning microscopy in frying of potato products. Microscopy and Analysis $1999 ; 37: 21-2$

Pedreschi F, Hernandez P, Figueroa C, Moyano P. Modeling water loss during frying of potato slices. International Journal of Food Properties $2005 ; 8$ : 289-99.

Pinthus EJ, Saguy IS. Initial interfacial tension and oil uptake by deep-fat fried foods. J Food Sci $1994 ; 59$ : 804-807, 823.

Pinthus EJ, Weinberg P, Saguy IS. Oil uptake in deep fat frying as affected by porosity. J Food Sci $1995 ; 60$ : 767-9.
Rajkumar V, Moreira R, Barrufet M. Modeling the structural changes of tortilla chips during frying. J Food Eng 2003 ; 60 : 167-75.

Rimac-Brncic S, Lelas V, Rade D, Simundic B. Decreasing of oil absorption in potato strips during deep fat frying. J Food Eng 2004 ; 64 : 237-41.

Rojas J, Avallone S, Brat P, Trystram G, Bohuon P. Effect of deep-fat frying on ascorbic acid, carotenoids and potassium contents of plantain cylinders. Int J Food Sci Nutr 2006 ; 57 : 123-36.

Saguy IS, Pinthus EJ. Oil uptake during deepfat frying: factors and mechanism. Food Technology $1995 ; 49$ : 142-5.

Seppanen CM, Saari Csallany A. Formation of 4- hydroxynonenal, a toxic aldehyde, in soybean oil at frying temperature. J Am Oil Chem Soc 2002 ; 79 : 1033-8.

Talburt WF. Frozen French Fries and Other Frozen Products. Westport : AVI. 1987.

Tan KJ, Mittal GS. Physicochemical properties changes of donuts during vacuum frying. International Journal of Food Properties 2006 ; $9: 85-98$.

Thanatuksorn $\mathrm{P}$, Pradistsuwana $\mathrm{C}$, Jantawat $P$, Suzuki T. Effect of surface roughness on post-frying oil absorption in wheat flour and water food model. J Sci Food Agric 2005 ; 85 : 2574-80.

Topin F, Tadrist L. Analysis of transport phenomena during the convective drying in superheated steam. Drying Technology 1997 ; 15 : 2239-61.

Tseng YC, Moreira R, Sun X. Total frying-use time effects on soybean-oil deterioration and on tortilla chip quality. Int / Food Sci Technol 1996 ; 31 : 287-94.

Ufheil G, Escher F. Dynamics of oil uptake during deep-fat frying of potato slices. Lebensmittel-Wissenschaft und Technologie $1996 ; 29$ : 640-4.

Vitrac O. Caractérisation expérimentale et modélisation de l'opération de friture. Massy, France: Ecole Nationale Supérieure des Industries Agricoles et Alimentaires, Ph.D. Thesis, 2000.

Yamsaengsung R, Moreira RG. Modeling the transport phenomena and structural changes during deep fat frying: Part I: Model development. J Food Eng 2002 ; 53 : 1-10.

Ziaiifar A, Achir N, Courtois F, Trezzani I, Trystram G. Review of mechanisms, conditions, and factors involved in the oil uptake phenomenon during the deep-fat frying process. Int / Food Sci Technol 2008 ; 43 : 1410-23.

Ziaiifar A, Courtois F, Trystram G. Porosity development and its effect on oil uptake during frying process. J Food Proc Eng 2010 ; $33: 191-212$. 